\title{
A 3D Facial Expression Database For Facial Behavior Research
}

\author{
Lijun Yin Xiaozhou Wei Yi Sun JunWang Matthew J. Rosato \\ Department of Computer Science, State University of New York at Binghamton
}

\begin{abstract}
Traditionally, human facial expressions have been studied using either $2 D$ static images or $2 D$ video sequences. The 2D-based analysis is incapable of handing large pose variations. Although $3 D$ modeling techniques have been extensively used for $3 D$ face recognition and $3 D$ face animation, barely any research on $3 D$ facial expression recognition using $3 D$ range data has been reported. A primary factor for preventing such research is the lack of a publicly available $3 D$ facial expression database. In this paper, we present a newly developed $3 D$ facial expression database, which includes both prototypical $3 D$ facial expression shapes and 2D facial textures of 2,500 models from 100 subjects. This is the first attempt at making a $3 D$ facial expression database available for the research community, with the ultimate goal of fostering the research on affective computing and increasing the general understanding of facial behavior and the fine $3 D$ structure inherent in human facial expressions. The new database can be a valuable resource for algorithm assessment, comparison and evaluation.
\end{abstract}

\section{Introduction}

Computer facial expression analysis would be highly beneficial for many fields including those as diverse as human computer interaction, security, medicine, behavior science, communication, and education. Currently, all existing face expression analysis and recognition systems rely primarily on static images or dynamic videos from many 2D facial expression databases (e.g., [19] and Table 1). Although some systems have been successful, the performance degradation remains when handling expressions with large head rotation, subtle skin movement, and/or lighting change with varying postures. In order to mitigate the problems inherent in the $2 \mathrm{D}$ based analysis, we propose to establish a new 3D facial expression database, and conduct facial expression analysis in a $3 \mathrm{D}$ space by exploring the surface information, which is beyond the availability from the 2D plane. In the following section, we will review the existing work, identify the critical issues to show why analyzing facial expression in a fully $3 \mathrm{D}$ space is necessary.

\subsection{The State of The Art}

Research on automatic techniques for analyzing human facial behavior has been conducted for over three decades $[11,32,34]$. There are two general approaches which have been developed relying on either $2 \mathrm{D}$ information or partial 3D information.

The conventional methods for facial expression recognition focuses on extracting the expression data needed to describe the change of facial features, such as Action Units (AUs) which are defined in the Facial Action Coding System (FACS) [11]. A number of techniques were successfully developed using 2D static images or video sequences, including machine vision techniques $[44,12,10$, $21,4,41,42]$ and machine learning techniques [1, 20, 5, 6, 45]. The excellent review of recent advances in this field can be found in $[27,42,46,28,15]$.

Recently, some researchers have noticed the importance of exploring 3D information to improve facial expression recognition. Some have successfully used partial 3D information, such as multiple views [29] or 3D models for facial expression analysis $[2,17,43,26]$. These methods are based on 2D images. They can alleviate the problems caused by different head poses to a certain degree with the assistance of a 3D model or with multiple views of the face. However, since no complete 3D individual facial geometric shapes are employed, the ability to handle large head pose variation and the ability to differentiate subtle expressions is inherently limited.

To the best of our knowledge, little investigation has been conducted on analyzing facial behavior in a complete $3 \mathrm{D}$ space even though it is believed to be a better reflection of facial behavior. In the following section, we summarize several critical issues and limitations of the existing facial expression recognition systems, and show the advantage of 3D facial expression analysis.

\subsection{Why 3D: Critical Issues and Limitations of 2D}

\section{(1) 3D surface features exhibited in facial expressions}

The common theme in the current research on face expression recognition is that the face is a flat pattern, like a 2D geometric shape associating with certain textures. This view has the consequences that expression variations is considered only in terms of measurements made on the picture plane. However, the common feature of faces is the three-dimensional surface rather than a two-dimensional pattern. Understanding the face as a mobile, bump surface instead of a flat pattern may have a theoretical implication as well as practical applications. Psychological research shows that the human visual system can perceive and understand embedded features contained in the 3D facial surface even when such features are not exhibited in corresponding 2D 
plane images. It is possible that the viewer actually represents the surface shape of the face when constructing representations for recognition [3]. This explains why human recognition of $2 \mathrm{D}$ facial expressions is presently so much better than machine recognition.

The facial expression is an entire facial behavior. Multidimensional expression space better characterizes this complexity [37]. Many expressions in this space exhibit subtle in-depth skin motion. For example, the skin extrusion in the areas of the cheek, forehead, glabella (in between eyebrows), nasolabial (in-between nose-side and mouth corners), crowfeet (out-corners of eyes), chin or mouth exhibits these subtle motions. These areas contain a high number of precious surface features (e.g., convex, concave, or other 3D primitive features), and could play a critical role in distinguishing subtle facial expressions. However, the 2D based approaches are hard to detect 3D surface features and in-depth motions (e.g., wrinkles) although they are good at detecting high-contrast features in salient organ areas (such as eye, nose, mouth).

Due to the limitations in describing facial surface deformation when 3D features are evaluated in 2D space, 2D images with a handful of feature units may not accurately reflect the authentic facial expressions. Therefore, there is a great demand for representing facial expressions in a 3D space in order to scrutinize the facial behavior at the level of subtlety explored between humanhuman interactions. Such a 3D-based analysis approach could allow us to examine the fine structure change for universal and complex expressions.

\section{(2) Pose / Posture:}

People rarely express emotions without head motion or posture spontaneity. Nevertheless, current research on facial expression analysis primarily focuses on the frontal view of face images, with very limited head motion or posture change. The assumption of frontal view expressions is not only unrealistic, but also jeopardizes the accurate expression analysis because head pose and posture are important cues, which, in conjunction with facial action, reflect a person's real emotion [28]. Large head pose change will cause an illumination change on the face which may cause part of the face to become invisible. The head motion and resulting occlusion increase the difficulty to track facial features and pose (e.g., in-depth direction) accurately and reliably in the $2 \mathrm{D}$ plane, jeopardizing the robust detection of AUs. Capturing 3D head orientation and analyzing facial expressions in 3D space has the potential to alleviate these problems related to pose and posture.

\section{(3) Benchmark 3D facial expression database}

A common testing resource is essential for research on facial expression analysis. Although there are a number of popular 2D face expression databases accessible for facial expression research, as of yet, no readily accessible database of test materials for 3D expression analysis has been established (see Table 1). The lack of an accredited common database (like the FERET and FRGC databases for face recognition) and evaluation methodology makes it difficult to compare, validate, resolve, and extend the issues concerned with 3D facial expression analysis. Currently, a number of standard face databases, containing both $2 \mathrm{D}$ and $3 \mathrm{D}$ data (e.g., $[30,13,14]$ ), are available to the face recognition community. However, these databases were not designed systematically for the purpose of facial expression recognition. They do not include either a whole set of prototypic expression data or 3D face expressions at various levels of intensity, therefore are not sufficient for $3 \mathrm{D}$ face expression research.

\begin{tabular}{|l|l|l|}
\hline $\begin{array}{l}\text { Data- } \\
\text { Base }\end{array}$ & \multicolumn{1}{|c|}{$\begin{array}{c}\text { Face } \\
\text { Recognition }\end{array}$} & $\begin{array}{l}\text { Face Expression } \\
\text { Recognition }\end{array}$ \\
\hline 2D & FERET [31], FRGC [30], & Cohn-Kanade [19], \\
& JAFFE [21], MMI [8], \\
& CMU-PIE [39], BioID[50] & RU-FACS-1 [9], \\
& AR [48], Yale [47], & Ekman-Hager [16,10] \\
& xm2vtsdb [18] & USC-IMSC [23], \\
& UT-Dallas [25, 24], & UT-Dallas [25, 24], \\
& Many others [46], .. & UA-UIUC [38], \\
\hline 3D [33], PICS[49],... \\
\hline & 3D FRGC [30, 22], & None \\
& DARPA-HumanID [14], & \\
& PRISM-ASU [13], & \\
& xm2vtsdb [18], .. & \\
\hline
\end{tabular}

Table 1. Survey of existing databases for research on face recognition and face expression recognition

In short, the establishment of a 3D facial expression database is crucial to enhancing facial behavior research. The lack of such essentials has impeded research in this area. In the following sections, we will introduce the database creation process and the organization of the database. We will also describe the process for data validation and assessment. Finally, the limitation and future extension of the current database are addressed.

\section{Creation of 3D Facial Expression Database}

\subsection{Capturing 3D Facial Expressions}

The development of our database was designed to sample facial behaviors with seven universal emotional states. Each expression is represented by multiple intensities which reflect different levels of spontaneity.

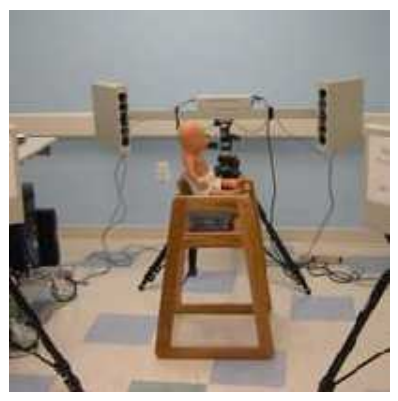

Figure 1. 3D face imaging system setup 


\section{(1) 3D face digitizer}

The $3 \mathrm{D}$ facial range data is captured with a $3 \mathrm{D}$ face imaging system (3DMD digitizer, Figure 1) [36] using a random light pattern projection in the speckle projection flash environment. The system projects a random light pattern onto the subject and captures his/her shape using the precisely synchronized digital cameras which are set at various angles in an optimum configuration. The six digital cameras and two light pattern projectors are positioned on two sides (three cameras and one projector on each side). The system automatically merges all six synchronized cameras' viewpoints data and produces a single 3D polygon surface mesh. Using the stereo photogrammetry technique, the 3D face surface geometry and surface texture are acquired. Each instant shot (less than 2 milliseconds capture time) outputs a set of data, including a pair of textures with two angle views and a wire-frame model. The texture size of the two-views image is around 1300 by 900 pixels. The model resolution is in the range from 20,000 polygons to 35,000 polygons, depending on the size of subject's face.

\section{(2) Expression scanning at work}

Each subject is instructed to sit in front of the 3D face capture system. They are requested to perform seven universal expressions, i.e., neutral, happiness, surprise, fear, sadness, disgust, and angry. Although the 3D capture system does not capture facial expressions dynamically, we require the subjects to perform each expression for a short period of time. The scan fires four instant shots to capture the four different degrees of the expression. The intensity ranges from low, middle, high, and highest, and the capturing at approximately ten-second intervals.

Ideally, a video clip could be used for eliciting the authentic expressions reflecting naturally occurring emotions. However, it is difficult to elicit a wide range of true emotions from a short video clip, especially for sadness and fear [38]. It is worth noting that archetypal emotions are a rare phenomenon. As quoted by Cowie et al [33], displays of intense emotion or "pure" primary emotions rarely happened.

The true emotion could be developed over a long time of involvement in special activities or events. The best elicitation could be from scenarios such as those shown in the reality TV shows, "Fear Factor", "Survivors", and "The Apprentice". However, it is not feasible to set up a lab environment to obtain such authentic and spontaneous expressions associated with true emotions. In everyday life, most people are likely to exhibit spontaneous emotions in a very light (low) intensity without exaggerated appearances. This common observation is similar to the scenario exhibited in the initial stage of the expression action. With this consideration, the subjects were asked to perform the light (low) intensity of each expression to simulate the spontaneity of the emotional state.

We requested each subject to perform four stages of expressions, ranging from low intensity, middle, high, and highest intensity of a specific expression. It was up to the subject to post four stages of expressions with his/her own style. Upon completing the face scanning, the data were annotated for archival as a ground truth.

\section{(3) Statistics of participants and expression data}

There were 100 subjects who participated in face scans, including undergraduates, graduates and faculty from our institute's departments of Psychology, Arts, and Engineering (Computer Science, Electrical Engineering and Mechanical Engineering). The majority of participants were undergraduates from the Psychology Department. The resulting database consists of about $60 \%$ female and $40 \%$ male subjects with a variety of ethnic/racial ancestries, including White, Black, East-Asian, Middle-east Asian, Hispanic Latino, and others.

Each subject performed seven expressions. With the exception of the neutral expression, each of the six prototypic expressions (happiness, disgust, fear, angry, surprise and sadness) includes four levels of intensity. Therefore, there are 25 instant 3D expression models for each subject, resulting in a total of 2,500 3D facial expression models in the database. Associated with each expression shape model, is a corresponding facial texture image captured at two views (about $+45^{\circ}$ and $-45^{\circ}$ ). As a result, the database consists of 2,500 two-view's texture images and 2,500 geometric shape models.

\subsection{Expression Data Description and Management}

The expression data includes the 3D model, texture, and enrollment information. Along with the raw model data, additional semantic and surface feature data are also archived. Figure 2 shows the data structure for archival. By query, the data is searchable by gender, ethnicity, expression (emotion state), and intensity.

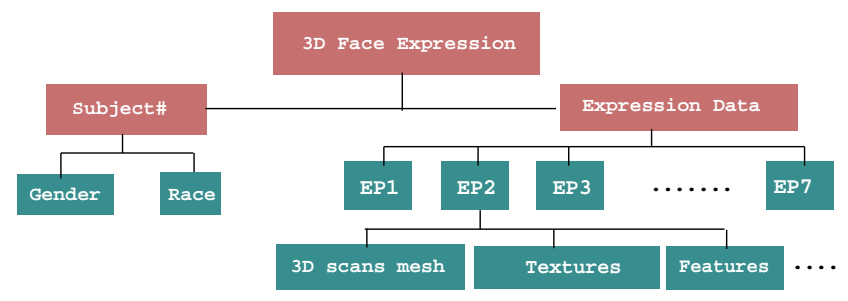

Figure 2. Data structure of 3D facial expressions for archival

\section{(1) Data processing}

In order to make the database useful for assessing and comparing algorithms using 2D-based and 3D-based facial expression recognition techniques, we provide both facial texture images and facial shape models as the raw data in the database.

Since the raw geometric models contain the unprocessed head-shoulder boundaries including necks and clothing, which are not "clean", further processing was performed to 
make the data easier to use. The original raw data was processed by truncating the boundary to generate a face model with the pure face region. The cropped face region contains about 13,000 - 21,000 polygons. In addition, a frontal view texture (512 by 512 pixels) is generated using our 3D face shape processing and warping tool. Therefore, in total, the database is composed of 2,500 raw 3D expression models, 2,500 raw textures in two-views' faces, 2,500 cropped models and 2,500 frontal view textures of the face regions.

In addition to the geometric data and texture data, a set of associated descriptors is also generated as an optional data set.

\section{(2) Associated Optional Descriptor}

(a) Feature point set: We picked 83 feature vertices on each facial model (Figure 4 (row 1)). Given the set of feature points on the face model labeled, the feature regions on the face surface can be easily determined. These features could be used as a ground truth to assess algorithms for $3 \mathrm{D}$ model segmentation and 3D feature detection.

(b) $3 D$ face pose: The obtained models contain various poses. We provide the model orientation using a normal vector with respect to the frontal projection plane. Given three vertices picked from two eye corners and a nose center, a triangle plane is formed. The norm of this plane represents the original face pose. The database includes such data for pose-related algorithm assessment.

\begin{tabular}{|l|l|}
\hline Raw data (Figure 3) & Produced data (Figure 4) \\
\hline 2,500 face shape models & $\begin{array}{l}\text { 2,500 cropped face } \\
\text { regional shape models }\end{array}$ \\
\hline $\begin{array}{l}\text { 2,500 face textures } \\
\text { (two views) }\end{array}$ & $\begin{array}{l}\text { 2,500 frontal texture of } \\
\text { facial regions }\end{array}$ \\
\hline & $\begin{array}{l}2,500 \text { data sets of } \\
\text { facial feature points }\end{array}$ \\
\hline & $\begin{array}{l}\text { 2,500 data sets of the } \\
\text { original facial poses }\end{array}$ \\
\hline
\end{tabular}

Table 2. Summary of the archived data including the raw data and the processed data.

In summary, the amount of 3D facial expression data archived in the database is listed in the Table 2. Note that since the database is designed to be available to public research, researchers in different areas can test their algorithms against the database and update or expand the dataset by adding new features in the future.

\section{Validation and Evaluation of the Database}

The quality of the $3 \mathrm{D}$ face expression database is evaluated through the validation experiments. The validation study addresses the question of whether the interpretations by machines are equal to those given by observers or performers. To do so, we conducted an analysis and test against our 3D expression database. Each expression data set was analyzed three times. Firstly, by the subject who performed the expression (as ground truth). Secondly, by observers from the Psychology Department who are experts in interpreting facial expressions (as expert votes). Thirdly, using machines via our facial expression recognizer (as machine votes). The following sub-sections report the statistical results of the expert evaluation and computer recognition.

\subsection{Subjective votes by observers}

As described in Section 2, the subjects provided the validation results for each expression with four intensities. Given such ground truth data, we compare the results by the subjective votes from two psychologists of Psychology Department. The confusion matrix is reported in the Table 3. The average expert recognition rate is $94.1 \%$ for low intensity expressions, $95.7 \%$ for middle intensity, $96.8 \%$ for high intensity, and $98.1 \%$ for highest intensity expressions. The most likely confused expressions were sad-fear and disgust-angry, even for experts.

\begin{tabular}{|l|l|l|l|l|l|l|l|}
\hline In/Out & Ang & Dis & Fea & Hap & Sad & Sur & Neu \\
\hline Anger & 94.9 & 2.5 & 1.2 & 0 & 0.3 & 0.2 & 0.9 \\
\hline Disgust & 2.6 & 95.4 & 0.9 & 0 & 0.9 & 0 & 0.2 \\
\hline Fear & 0.1 & 0.5 & 96.4 & 0 & 2.4 & 0.1 & 0.5 \\
\hline Happy & 0.1 & 0 & 0.1 & 99.4 & 0 & 0.4 & 0 \\
\hline Sad & 1.0 & 0.2 & 2.4 & 0 & 96.2 & 0 & 0.2 \\
\hline Surprise & 0.4 & 0 & 0.2 & 0.4 & 0 & 99.0 & 0 \\
\hline Neutral & 0.8 & 0 & 0.2 & 0 & 0.3 & 0 & 98.7 \\
\hline
\end{tabular}

Table 3. Confusion matrix of expert voting averagely for four intensities of expressions (\%).

\subsection{Objective votes by machine classification}

To validate the created 3D facial expression database, we conducted experiments on face expression recognition using our newly developed 3D face expression representation and classification algorithm. The basic algorithm is outlined as follows: (details in the report [7]).

Given the set of expression range models, in order to better characterize 3D features of the facial surface, each vertex on the individual model is labeled by one of the twelve primitive surface. Our labeling approach is based on the estimation of principal curvatures of the facial surface. It is believed that the curvature information is a good reflection of local shape of the facial surface [40].

In order to classify the facial expressions based on the 3D facial expression data, we segment the 3D face surface into seven local expressive regions (excluding interiors of mouth, interior of eyes and nose bridge), and conduct the histogram statistics on each region in terms of the twelve primitive surface label distribution. Each expressive region forms a twelve-dimension feature vector, in which each 
element is defined as a ratio of the number of vertices with a specific label type to the number of vertices in the local region. As such, an 84-dimension feature vector is constructed on the entire facial region. The facial expression surface labels exhibit different patterns which correspond to different facial expressions. Such feature vectors are used for expression classification.

We conducted facial expression recognition using pure 3D geometric shape models from our 3D facial expression database. The experiment is person-independent, which means the query subject has never appeared in the training set. We applied linear discriminant analysis (LDA) classifier to classify the prototypic facial expressions of sixty subjects. The correct recognition rate is about $83.6 \%$.

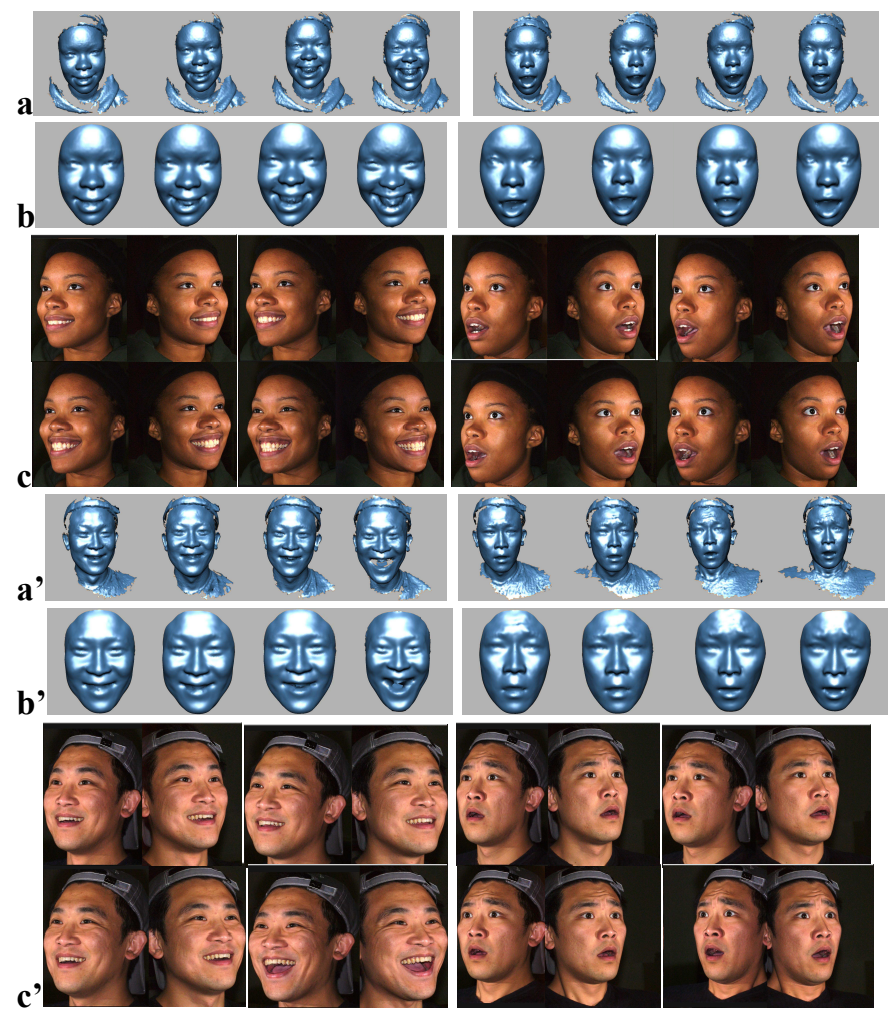

Figure 3: Sample expressions: Left four (happiness) and right four (surprise) with four levels of intensity. a-a' are raw models; $b$-b' are cropped shape models in face regions. c-c' are two views' textures.

\section{Limitation and Development of Database}

There are several limitations in the current version of the database in terms of dynamics, FACS-related coding and the expression variety in the expression space. Limited by the speed of 3D imaging capture system and post processing load, no 3D dynamic expressions are captured in the current version. The number of expression types is still limited to the prototypic expression space, more spontaneous expressions need to be included for naturally occurring emotion analysis. Our future work will focus on the following aspects:

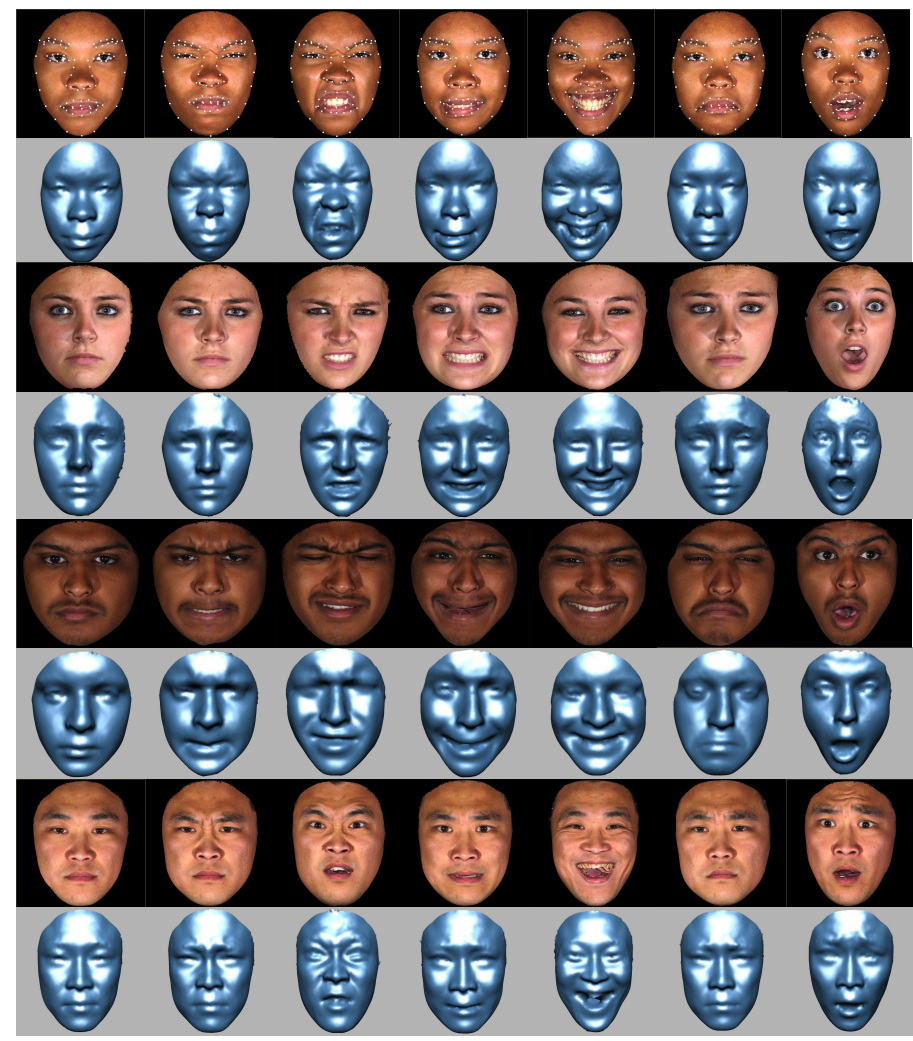

Figure 4: Four sample subjects showing seven expressions (neutral, angry, disgust, fear, happiness, sadness, and surprise). The facial shape model and frontal-view textures are produced. The first row shows a sample set of the picked feature points.

(1) Dynamics: We will extend the database to include dynamic 3D facial expression sequences using a real-time dynamic range system, with a super-high resolution model representation. As such, the 3D action units coding and labeling could be further explored.

(2) Expression space: We will include more spontaneous 3D expression data with more affects states (such as boredom, skepticism, shame, etc.) through eliciting children/adults emotion response with the experiments designed and guided by our collaborated psychologists.

(3) Applications in medical and psychological research: We will be interested in the study of the clinically interested data for diagnosis purpose. For example, reading pain expressions when the self-report is not possible for people like non-communicative adults, developmentally delayed children or newborns. We will also extend the 3D facial expression database to the emerging field of applications, such as using $3 \mathrm{D}$ expression models as a source of stimuli for the psychological research to diagnose, assess and rehabilitate 
patients with brain or psychological disorders (e.g., alzheimer, etc.) [35].

\section{Conclusion}

We have developed a new 3D facial expression database for the scientific research community. This is the first attempt to foster the research on analyzing the facial behavior in a complete 3D space, targeting the identification of more detail and subtle facial behavior. The future challenge is to further develop the $3 \mathrm{D}$ facial expression database using the dynamic and spontaneous 3D high-resolution expression data in order to move closer to developing a naturally occurring facial behavior analysis system.

\section{Acknowledgement}

This material is based upon the work supported in part by the National Science Foundation under grants IIS0541044, IIS-0414029, and the NYSTAR's James D. Watson Investigator Program. We would like thank Gina Shroff, Peter Gerhardstein, Joseph Morrissey of the Department of Psychology, Lee Serversky and Ben Myerson of the Computer Science Department for the help during the process of creating the database.

\section{References}

[1] M. Bartlett, J. Hager, P. Ekman, and T. Sejnowski. Measuring facial expressions by computer image analysis. Psychophysiology, 36, 1999.

[2] B. Braathen, M. Bartlett, G. Littlewort, et al. An approach to automatic recognition of spontaneous facial actions. FGR 2002.

[3] V Bruce, M. Burton, and T. Doyle. Faces as surfaces. Processing Images of Faces, 1992.

[4] Y Chang, C. Hu, and M. Turk. Probabilistic expression analysis on manifolds. CVPR'04, Washington DC, 2004.

[5] I. Cohen, F. Cozman, N. Sebe, M. Cirelo, and T. Huang. Semisupervised learning of classifiers: Theory, algorithms for Bayesian network classifiers and application to human-computer interaction. IEEE Trans. PAMI, 26(12), 2004.

[6] I. Cohen, N. Sebe, A. Garg, L. Chen, and T. Huang. Facial expression recognition from video sequences: temporal and static modeling. CVIU, 91(1), 2003

[7] J. Wang, L. Yin, et al, "3D facial expression recognition based on primitive surface feature distribution", Tech. Report, Binghamton U, 2006. [8] Man machine interaction group. http://www.mmifacedb.com/. Delft University of Technology, 2005.

[9] RU-FACS-1 Database. http://mplab.ucsd.edu/databases/databases.html. [10] G. Donato, M. Bartlett, J. Hager, P. Ekman, and T. Sejnowski. Classifying facial actions. IEEE Trans. PAMI, 21(10):974-989, 1999.

[11] P. Ekman and W. Friesen. Facial Action Coding System. New York: Consulting Psychologists Press, 1977.

[12] I. Essa and A. Pentland. Coding, analysis, interpretation, and recognition of facial expressions. IEEE Trans. PAMI, 19(7), 1997.

[13] PRISM-ASU. http://prism.asu.edu/3dface/default.asp.

[14] USF DARPA Human ID 3D face database. Courtesy of Prof. Sudeep Sarkar, University of South Florida, Tampa, FL.

[15] B. Fasel and J. Luettin. Automatic facial expression analysis: A survey. Pattern Recognition, 36(1), 2003.

[16] W. Friesen and P. Ekman. Dictionary - Interpretation of FACS Scoring. Unpublished manuscript, UC San Francisco, 1987.
[17] S. Gokturk, J. Bouguet, C. Tomasi, and B. Girod. Model-based face tracking for view-independent facial expression recognition. In FGR 2002. [18] K Messer, J Matas, J Kittler, et al. Xm2vtsdb: The extended m2vts database. In International Conference of AVBPA, March 1999.

$\mathrm{http}: / /$ www.ee.surrey.ac.uk/Research/VSSP/xm2vtsdb/

[19] T. Kanade, J.Cohn, and Y. Tian. Comprehensive database for facial expression analysis. FGR'00, France, 2000.

[20] G. Littlewort, M. Bartlett, I. Fasel, J. Susskind, and J. Movellan. Dynamics of facial expression extracted automatically from video. In CVPR Workshop on FPIV'04, 2004.

[21] M. Lyons, et al. Automatic classification of single facial images. IEEE Trans. PAMI, 21(12):1357-1362, 1999.

[22] K. Chang and K. Bowyer and P. Flynn. An evaluation of multimodal 2D+3D face biometrics, IEEE Trans. on PAMI. 27(4): 619-624. 2005.

[23] U. Neumann. Facial expression analysis and synthesis (NSF report). http://imsc.usc.edu/research/project/facialexp/.

[24] A. O'Toole. Psychological and neural perspectives in human face recognition. In The Handbook of Face Recognition, 2004, Springer-Verlag. Editors: S. Li and A. Jain.

[25] A. O'Toole, J. Harms, et al. A video database of moving faces and people. IEEE Trans. PAMI, 27(5), 2005.

[26] L. Zalewski and S. Gong. Synthesis and recognition of facial expressions in virtual 3D views. In FGR'04, 2004.

[27] M. Pantic and L. Rothkrantz. Automatic analysis of facial expressions: the state of the art. IEEE Trans. PAMI, 22(12), 2000.

[28] M. Pantic and L. Rothkrantz. Toward an affect-sensitive multimodal human-computer interaction. Proceedings of IEEE, 91(9):1370-1390, 2003.

[29] M. Pantic and L. Rothkrantz. Facial action recognition for facial expression analysis from static face images. IEEE Trans. on SMC Part B: Cybernetics, 34(3):1449-1461, 2004.

[30] P. Phillips, P. Flynn, T. Scruggs, K. Bowyer, J. Chang, K. Hoffman, J. Marques, J. Min, and W. Worek. Overview of the face recognition grand challenge. CVPR05, San Diego, CA, 2005.

[31] P. Phillips, H. Moon, P. Rauss, et al., The FERET evaluation methodology for face recognition algorithm. IEEE Trans. PAMI, 22 (10), 2000.

[32] R. Picard. Affective computing: challenges. Inter. Journal of Human Computer Studies, 59(1-2):55-64, 2003.

[33] E. Douglas-Cowie, R. Cowie and M. Schroder. A new emotion database: considerations, sources and scope. Proc. of the ISCA ITRW on Speech and Emotion, Newcastle, 2000, pp. 39-44.

[34] R.Cowie, E. Douglas-Cowie, et al. Emotion Recognition in Human Computer Interaction. IEEE Signal Processing Magazine 18 (1). 2001.

[35] A. Rizzo. Virtual reality and disability: emergence and challenge. Disability and Rehabilitation, 24(11), 2002.

[36] 3DMD Inc., http://www.3dmd.com, 2005.

[37] J. Russell. Is there universal recognition of emotion from facial expression? Psychological Bulletin, 115(1):102-141, 1994.

[38] N. Sebe, M. Lew, I. Cohen, Y Sun, T. Gevers, and T. Huang. Authentic facial expression analysis. In FGR 2004.

[39] T. Sim, S. Baker, and M. Bsat. The CMU pose, illumination and expression database. IEEE Trans. PAMI, 25(12), 2003.

[40] H. Tanaka, M. Ikeda, and H. Chiaki. Curvature-based face surface recognition using spherical correlation. In FGR'1998.

[41] F. Bettinger and T.F.Cootes. A Model of Facial Behavior. In FGR'04. [42] Y Tian, T. Kanade, and J. Cohn. Recognizing action units for facial expression analysis. IEEE Trans. on PAMI, 23(2), 2001.

[43] Z. Wen and T. Huang. Capturing subtle facial motions in 3D face tracking. In IEEE Inter. Conf. on Computer Vision, 2003.

[44] Y. Yacoob and L. Davis. Recognizing human facial expressions from long image sequences using optical flow. IEEE Trans. PAMI, 18 (6), 1996.

[45] Y Zhang and Q. Ji. Active and dynamic information fusion for facial expression understanding from image sequences. IEEE Trans. PAMI, 27 (5):699-714, May 2005.

[46] W. Zhao, R. Chellappa, P. Phillips, and A. Rosenfeld. Face recognition: A literature survey. ACM Computing Surveys, 35(4), 2003.

[47] http://cvc.yale.edu/projects/yalefaces/yalefaces.html

[48] http://rvl1.ecn.purdue.edu/ aleix/aleix_face_DB.html

[49] PICS database, http://pics.psych.stir.ac.uk/index.html

[50]http://www.humanscan.de/support/downloads/facedb.php 